\title{
An Explicit Formulation for Two Dimensional Vector Partition Functions
}

\author{
ZHIQIANG XU
}

\begin{abstract}
In this paper, an explicit formulation for two dimensional multivariate truncated power functions is presented, and a simplified explicit formulation for two dimensional vector partition functions is given. Moreover, Popoviciu's formulation for restricted integer partition functions is generalized and the generalized Frobenius problem is also discussed.
\end{abstract}

\section{Introduction}

The vector partition function that we are interested in is in the form of

$$
t(\mathbf{b} \mid M)=\#\left\{\mathbf{x} \in \mathbf{Z}_{+}^{n} \mid \mathbf{M} \mathbf{x}=\mathbf{b}\right\},
$$

where, $\mathbf{Z}_{+}$denotes the nonnegative integers, $\mathbf{M}$ is a fixed $s \times n$ integer matrix with columns $m_{1}, \cdots, m_{n} \in \mathbf{Z}^{s}$ and $\mathbf{b}$ is a variable vector in $\mathbf{Z}^{s}$. To guarantee that $t(\mathbf{b} \mid M)$ is finite, we require $\left[\left\{m_{1}, \cdots, m_{n}\right\}\right]$ does not contain the origin, where $[A]$ denotes the convex hull of a given set $A$. The vector partition function $t(\mathbf{b} \mid M)$, which is also called a discrete truncated power, has many applications in various mathematical areas including Algebraic Geometry [26], Representation Theory[29], Number Theory [23], Statistics[16] and Randomized Algorithm [32] among others.

When $s=1$, an explicit formulation for $t(\mathbf{b} \mid M)$, which counts the integer solutions for the linear Diophantine equation, is presented in [1]. Especially, Popoviciu gave a beautiful and surprising formulation for $t(n \mid M)([\mathbf{2 7}])$, when $M=(a, b)$ where $a$ and $b$ are relatively prime.

For the general matrix $M$, the nature of $t(\mathbf{b} \mid M)$ is investigated and the piecewise structure of $t(\mathbf{b} \mid M)$ is given in [15] and [31]. Moreover, one is also interested in the explicit formulation of $t(\mathbf{b} \mid M)$. For the general matrix $M$, a powerful method for obtaining $t(\mathbf{b} \mid M)$ is described in $[\mathbf{8}, \mathbf{3 0}]$. Another interesting algorithm for computing $t(\mathbf{b} \mid M)$ as a function of $\mathbf{b}$ is also introduced in [3]. When $M$ is unimodular, where every nonsingular square submatrix has determinant \pm 1 , two algebraic algorithms for generating the explicit formulation for $t(\mathbf{b} \mid M)$ is presented in [17]. However, all of these methods depend on complex computation. In [34], based on multivariate truncated power functions $T(\mathbf{x} \mid M)$, an explicit formulation for $t(\mathbf{b} \mid M)$ is presented. However, the formulation involves multivariate truncated power functions $T(\mathbf{x} \mid M)$,

1991 Mathematics Subject Classification. Primary 41A15, 05A17; Secondary 11D04,52B20.

Key words and phrases. Multivariate truncated powers, Vector partition function.

Project Supported by The National Natural Science Foundation of China(10401021). 
which are not in explicit form, and high-dimensional Fourier-Dedekind sums, so we have to give an explicit form for $T(\mathbf{x} \mid M)$ and simplify high-dimensional FourierDedekind sums, in order to predigest the explicit formulation for $t(\mathbf{b} \mid M)$. The goal of this paper is to generalize Popoviciu's formulation, and give a simplified explicit formulation for two dimensional vector partition functions. We believe that the results in this paper are not only helpful for understanding high dimensional vector partition functions but also useful for solving two linear Diophantine equations $[18,35]$.

The rest of the paper is organized as follows. To help make this paper selfcontained we shall first introduce notations and definitions in Section 2. In Section 3 , we recall previous results regarding vector partition functions $t(\mathbf{b} \mid M)$. Section 4 generalizes the Popoviciu's formulation to two dimension. In Section 5, the generalized Frobenius problem is investigated. Finally, Section 6 gives an explicit formulation for multivariate truncated powers in the case where $s=2$ and show that a high-dimensional Fourier-Dedekind sum can be converted to a one-dimensional Fourier-Dedekind sum, which is convenient for computing. A simplified explicit formulation for two-dimension vector partition functions is then given.

\section{Preliminaries}

To describe the nature of $t(\mathbf{b} \mid \mathbf{M})$, we introduce several notations and definitions in which the common terminology of multiset theory is adopted. Intuitively, a multiset is a set with possible repeated elements; for instance $\{2,2,2,3,4,4\}$. Let $Y$ be an $s \times n$ matrix and $Y$ can be considered as a multiset of elements of $\mathbf{R}^{s}$. The cone spanned by $Y$, denoted by cone $(Y)$, is the set

$$
\left\{\sum_{y \in Y} a_{y} y: a_{y} \geq 0 \text { for all } y\right\} .
$$

Denote by $\operatorname{cone}^{\circ}(Y)$ the relative interior of cone $(Y)$. Let $\mathcal{Y}(M)$ denote the set consisting of those submultisets $Y$ of $M$ for which $M \backslash Y$ does not span $\mathbf{R}^{s}$. Let the set $c(M)$ be the union of all sets $\operatorname{span}(M \backslash Y)$ as $Y$ runs over $\mathcal{Y}(M)$. A connected component of $\operatorname{cone}^{\circ}(M) \backslash c(M)$, is called a fundamental $M$-cone. For a fundamental $M$-cone $\Omega$, we set $v(\Omega \mid M):=\Omega-[[M))$. Here, $[[M)):=\left\{\sum_{j=1}^{n} a_{j} m_{j}: 0 \leq a_{j}<\right.$ $1, \forall j\}, \Omega-[[M))$ is the set of all elements of the form $a-b$, where $a \in \Omega$ and $b \in[[M))$.

We shall use the standard multiindex notation. Specifically, an element $\alpha \in \mathbf{N}^{m}$ is called an $m$-index, and $|\alpha|$ is called the length of $\alpha$. Define $z^{\alpha}:=z_{1}^{\alpha_{1}} \cdots z_{m}^{\alpha_{m}}$ for $z=\left(z_{1}, \cdots, z_{m}\right) \in \mathbf{C}^{m}$ and $\alpha=\left(\alpha_{1}, \cdots, \alpha_{m}\right) \in \mathbf{N}^{m}$. For $y=\left(y_{1}, \cdots, y_{s}\right) \in \mathbf{R}^{s}$ and a function $f$ defined on $\mathbf{R}^{s}$, we denote by $D_{y} f$ the directional derivative of $f$ in the direction $y$,i.e. $D_{y}=\sum_{j=1}^{s} y_{j} D_{j}$, where, $D_{j}$ denote the partial derivative with respect to the $j$ th coordinate. For $v:=\left(v_{1}, \cdots, v_{m}\right) \in \mathbf{N}^{m}$, we let $D^{v}=$ $D_{1}^{v_{1}} \cdots D_{m}^{v_{m}}$ and $v !=\prod_{i} v_{i}$ ! Moreover, we let $e:=(1,1, \cdots, 1) \in \mathbf{Z}^{s}$.

Let $\mathcal{S}_{k}(M)=\left\{Y \subseteq M: \# Y=s+k, \operatorname{span}(Y)=\mathbf{R}^{s}\right\}$ and $\mathcal{B}(Y)=\{X \subseteq Y$ : $\left.\# X=s, \operatorname{span}(X)=\mathbf{R}^{s}\right\}$. If for any $Y \in \mathcal{S}_{k}(M), \operatorname{gcd}\{|\operatorname{det}(X)|, X \in \mathcal{B}(Y)\}=1$, then $M$ is called a $k$-prime matrix. In particular, when $M$ is an 1 -prime matrix, $M$ is also called a pairwise relative prime matrix. When $s=1, k$-prime matrix means that no $k$ of the integers $m_{1}, m_{2}, \cdots, m_{n}$ have a common factor, where $m_{i}, i=1, \cdots, n$ are the elements in $M$. Moreover, we denote $e^{\frac{2 \pi i}{k}}$ by $W_{k}$. 
The multivariate truncated power $T(\cdot \mid M)$ associated with $M$, which was introduced by W.Dahmen [10] firstly, is the distribution given by the rule

$$
T(\cdot \mid M): \phi \mapsto \int_{\mathbf{R}_{+}^{n}} \phi(M u) d u, \phi \in \mathcal{D}\left(\mathbf{R}^{s}\right),
$$

where $\mathcal{D}\left(\mathbf{R}^{s}\right)$ is the space of test functions on $\mathbf{R}^{s}$,i.e. the space of all compactly supported and infinitely differentiable functions on $\mathbf{R}^{s}$. In fact, $T(\cdot \mid M)$ agrees with some homogeneous polynomial of degree $n-s$ on each fundamental $M$-cone. When $M$ is an $s \times s$ invertible matrix, $T(\cdot \mid M)$ agrees with the function on $\mathbf{R}^{s}$ which takes value $\frac{1}{[\operatorname{det}(M) \mid}$ on cone $(M)$ and 0 elsewhere.

In $[\mathbf{2 2}]$, an efficient method for computing the multivariate truncated power is presented.

TheOREM 2.1. ([22]) Let $M$ be an $s \times n$ matrix with columns $m_{1}, \cdots, m_{n} \in$ $\mathbf{Z}^{s} \backslash\{0\}$ such that the origin does not contain in $\operatorname{conv}(M)$. For any $\lambda_{1}, \cdots, \lambda_{n} \in \mathbf{R}$, and $\mathbf{x}=\sum_{j=1}^{n} \lambda_{j} m_{j}$,

$$
T(\mathbf{x} \mid M)=\frac{1}{n-s} \sum_{j=1}^{n} \lambda_{j} T\left(x \mid M \backslash m_{j}\right) .
$$

For more detailed information about the function, the reader is referred to $[6],[10]$.

A multivariate Box spline $B(\cdot \mid M)$ associated with $M$ was introduced in [5] and $[6]$, which is the distribution given by the rule

$$
B(\cdot \mid M): \phi \mapsto \int_{[0,1)^{n}} \phi(M u) d u, \phi \in \mathcal{D}\left(\mathbf{R}^{s}\right) .
$$

By taking $\phi=\exp (-i y \cdot)$ in $(2.3)$, we obtain the Fourier transform of $B(\cdot \mid M)$ as

$$
\widehat{B}(\zeta \mid M)=\prod_{j=1}^{n} \frac{1-\exp \left(-i \zeta^{T} m_{j}\right)}{i \zeta^{T} m_{j}}, \zeta \in \mathbf{C}^{s} .
$$

For more detail information about Box splines, the reader is referred to $[\mathbf{7}]$.

REMARK 2.2. Our definition of a fundamental $M$-cone is slightly different from that presented in [15]. In [15], a fundamental $M$-cone is defined as a connected component of $\operatorname{cone} e^{\circ}(M) \backslash c(M)$, where $c(M)$ is the union of all sets $\operatorname{span}(M \backslash Y)$ as $Y$ runs over $\mathcal{Y}(M)$. In fact, the fundamental $M$-cone defined in this paper may be larger than the one defined in [15]. All the conclusions in [15], however, also hold for the larger fundamental $M$-cone. Prof. M. Vergne introduced this new definition of a fundamental $M$-cone in a private communication.

\section{Vector partition functions}

To describe the nature of $t(\mathbf{b} \mid M)$, we let $M_{\theta}:=\left\{y \in M: \theta^{y}=1\right\}$ and let $A(M):=\left\{\theta \in(\mathbf{C} \backslash\{0\})^{s}: \operatorname{span}\left(M_{\theta}\right)=\mathbf{R}^{s}\right\}$. Recall $e=(1,1, \cdots, 1) \in \mathbf{Z}^{s}$, as for any $y \in M, e^{y}=1, e \in A(M)$.

The following qualitative result about $t(\cdot \mid M)$ is presented in [15].

THEOREM 3.1. ([15]) Let $M=\left\{m_{1}, \cdots, m_{n}\right\}$ be a multiset of integer vectors in $\mathbf{R}^{s}$ such that $M$ spans $\mathbf{R}^{s}$ and the convex hull of $M$ does not contain the origin. Then for any fundamental $M$-cone $\Omega$, there exists a unique element $f_{\Omega}(\alpha \mid M)=$ 
$\sum_{\theta \in A(M)} \theta^{\alpha} p_{\theta, \Omega}(\alpha)$ such that $f_{\Omega}(\alpha \mid M)$ agrees with $t(\alpha \mid M)$ on $v(\Omega \mid M)$, where $p_{\theta, \Omega}(\cdot)$ is a polynomial with degree less than $\# M_{\theta}-s$.

An explicit formulation for $p_{e, \Omega}(\alpha)$, which is the polynomial part for $t(\alpha \mid M)$, is presented in the following theorem.

THEOREM 3.2. ([34]) Under the condition for Theorem 3.1, $p_{e, \Omega}(x)=\sum_{k=0}^{n-s} p_{k, \Omega}(x)$, where $p_{k, \Omega}(x)$ is homogeneous polynomial of degree $n-s-k$, defined inductively by $p_{0, \Omega}(x)=T(x \mid M), p_{k, \Omega}(x)=-\sum_{j=0}^{k-1}\left(\sum_{|v|=k-j} D^{v} p_{j, \Omega}(x)(-i)^{|v|} D^{v} \widehat{B}(0 \mid M) / v !\right), k \geq 1$, where, $x \in \Omega$.

More generally, an explicit formulation for $p_{\theta, \Omega}$ is also given as follows.

THEOREM 3.3. ([34]) Given $\theta_{0} \in A(M) \backslash e$, under the condition for Theorem 3.1, $p_{\theta_{0}, \Omega}(x)=\sum_{\mu=0}^{n-s-\kappa} p_{\mu, \Omega}^{\theta_{0}}(x)$, where $\kappa=\#\left(M \backslash M_{\theta_{0}}\right), p_{\mu, \Omega}^{\theta_{0}}(x)$ is homogeneous polynomial of degree $n-s-\kappa-\mu$, defined inductively by

$$
\begin{aligned}
& p_{0, \Omega}^{\theta_{0}}(x)=q_{0, r}^{\theta_{0}}(x), \\
& p_{\mu, \Omega}^{\theta_{0}}(x)=q_{\mu, r}^{\theta_{0}}(x)-\sum_{j=0}^{\mu-1}\left(\sum_{|v|=\mu-j} D^{v} p_{\mu, \Omega}^{\theta_{0}}(x)(-i)^{|v|} D^{v} \widehat{B}\left(0 \mid \widehat{M}_{r}\right) / v !\right), \mu \geq 1 .
\end{aligned}
$$

Here, $q_{\mu, r}^{\theta_{0}}(x)$ is a polynomial which is determined by the following conditions: when $x \in \Omega, q_{\mu, r}^{\theta_{0}}(x)=\sum_{j_{1}+\cdots+j_{\kappa}=\mu} \prod_{i=1}^{\kappa} \frac{s_{1+j_{i}}\left(\theta_{0}-m_{i}\right)}{\left(j_{i}+1\right) !} \frac{1}{r^{\kappa}} D_{m_{1}}^{j_{1}} \cdots D_{m_{\kappa}}^{j_{\kappa}} T\left(x \mid M_{\theta_{0}}\right)$, where $s_{0}(x)=$ $\frac{x-x^{r}}{x-1}, s_{j}(x)=x s_{j-1}^{\prime}(x), j \in \mathbf{Z}_{+}$.

In particular, when $M$ is a 1-prime matrix, a simple formulation for $t(\cdot \mid M)$ is shown in the following theorem.

THEOREM 3.4. [34] Under the condition for Theorem 3.1, when $M$ is a 1-prime matrix,

$$
f_{\Omega}(\alpha \mid M)=p_{e, \Omega}(\alpha \mid M)+\sum_{\theta \in A(M) \backslash e} \theta^{\alpha} \frac{1}{\left|\operatorname{det}\left(M_{\theta}\right)\right|} \prod_{w \in M \backslash M_{\theta}} \frac{1}{1-\theta^{-w}} 1_{\operatorname{cone}\left(M_{\theta}\right)}(\Omega),
$$

where $p_{e, \Omega}(\alpha \mid M)$ is given in Theorem 3.2.

For the convenience of description, throughout the rest of the paper, we suppose $M$ is a 1-prime matrix without further declaration. According to Theorem 3.4, to give a simple explicit formulation for $t(\mathbf{b} \mid M)$, we have to present an explicit formulation for $T(\mathbf{x} \mid M)$. Moreover, to calculate the elements in $A(M)$ is a non-trivial problem, and hence, we have to predigest the non-polynomial part for $t(\mathbf{b} \mid M)$.

\section{Generalized Popoviciu's formulation}

In this section, we are interested in $t(\mathbf{n} \mid M)$, where $M=\left(\begin{array}{lll}x_{1} & x_{2} & x_{3} \\ y_{1} & y_{2} & y_{3}\end{array}\right) \in$ $\mathbf{Z}^{2 \times 3}, \mathbf{n}=\left(n_{1}, n_{2}\right)^{T} \in \mathbf{Z}_{+}^{2}$. Without loss of generality, we suppose $\frac{y_{1}}{x_{1}}<\frac{y_{2}}{x_{2}}<$ $\frac{y_{3}}{x_{3}}$. Obviously, for the matrix $M$, there exist two fundamental $M$-cones, i.e. $\Omega_{1}=\left\{(x, y)^{T} \mid(x, y)^{T} \in \operatorname{cone}(M), \frac{y_{1}}{x_{1}}<\frac{y}{x}<\frac{y_{2}}{x_{2}}\right\}$ and $\Omega_{2}=\left\{(x, y)^{T} \mid(x, y)^{T} \in\right.$ cone $\left.(M), \frac{y_{2}}{x_{2}}<\frac{y}{x}<\frac{y_{3}}{x_{3}}\right\}$ (See Fig. 1). 


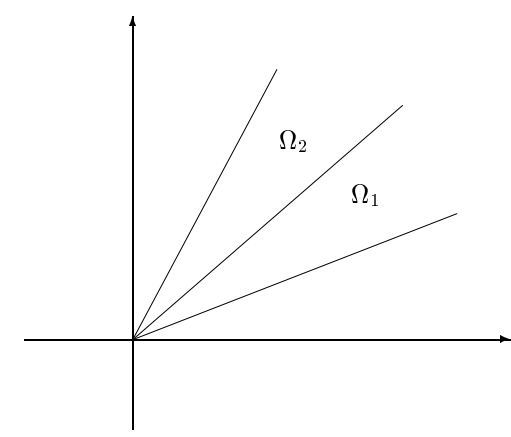

Fig. 1.The fundamental M-cones.

To describe conveniently, we let $M_{i j}=\left(\begin{array}{ll}x_{i} & x_{j} \\ y_{i} & y_{j}\end{array}\right)$, and let $Y_{i j}=\operatorname{det}\left(M_{i j}\right)$, where $i<j$. To describe the explicit formulation for $t(\mathbf{n} \mid M)$, we need to define the fractional part function $\{x\}$ which denotes the fractional part of $x$, i.e. $\{x\}=$ $x-\lfloor x\rfloor$.

In this section, our goal is to generalize the following beautiful formula due to Popoviciu:

THEOREM 4.1. $[\mathbf{2 7}]$ If $a$ and $b$ are relatively prime,

$$
t(n \mid(a, b))=\frac{n}{a b}-\left\{\frac{b^{-1} n}{a}\right\}-\left\{\frac{a^{-1} n}{b}\right\}+1,
$$

where $b^{-1} b \equiv 1 \bmod a$, and $a^{-1} a \equiv 1 \bmod b, n \in \mathbf{Z}_{+}$.

In order to generalize Theorem 4.1, we firstly consider the explicit formulation for $T(\mathbf{x} \mid M)$.

Lemma 4.2. Suppose the matrix $M=\left(\begin{array}{lll}x_{1} & x_{2} & x_{3} \\ y_{1} & y_{2} & y_{3}\end{array}\right) \in \mathbf{Z}^{2 \times 3}$. When $\mathbf{x}=$ $(x, y)^{T} \in \bar{\Omega}_{1}, T(\mathbf{x} \mid M)=\frac{y x_{1}-x y_{1}}{\left(x_{1} y_{2}-y_{1} x_{2}\right)\left(x_{1} y_{3}-y_{1} x_{3}\right)}$; when $\mathbf{x}=(x, y)^{T} \in \bar{\Omega}_{2}, T(\mathbf{x} \mid M)=$ $\frac{x y_{3}-y x_{3}}{\left(x_{2} y_{3}-y_{2} x_{3}\right)\left(x_{1} y_{3}-y_{1} x_{3}\right)}$.

Proof. Based on Theorem 2.1 and $T\left(\mathbf{x} \mid M_{i j}\right)=\frac{1}{\operatorname{det}\left(M_{i j}\right)}, \mathbf{x} \in \operatorname{cone}\left(M_{i j}\right), i<j$, the Lemma can be proved easily after a brief calculation.

The main theorem in this section is:

THEOREM 4.3. Suppose the 1-prime matrix $M=\left(\begin{array}{lll}x_{1} & x_{2} & x_{3} \\ y_{1} & y_{2} & y_{3}\end{array}\right)$. When $\mathbf{n}=$ $\left(n_{1}, n_{2}\right)^{T} \in \bar{\Omega}_{1} \cap \mathbf{Z}^{2}$,

$$
\begin{aligned}
t(\mathbf{n} \mid M) & =\frac{n_{2} x_{1}-n_{1} y_{1}}{Y_{12} Y_{13}}-\left\{\frac{\left(f_{12} Y_{13}+g_{12} Y_{23}\right)^{-1}\left(n_{2}\left(f_{12} x_{1}+g_{12} x_{2}\right)-n_{1}\left(f_{12} y_{1}+g_{12} y_{2}\right)\right)}{Y_{12}}\right\} \\
& -\left\{\frac{\left(f_{13} Y_{12}+g_{13} Y_{23}\right)^{-1}\left(n_{2}\left(f_{13} x_{1}+g_{13} x_{3}\right)-n_{1}\left(f_{13} y_{1}+g_{13} y_{3}\right)\right)}{Y_{13}}\right\}+1 ;
\end{aligned}
$$

when $\mathbf{n}=\left(n_{1}, n_{2}\right)^{T} \in \bar{\Omega}_{2} \cap \mathbf{Z}^{2}$,

$$
\begin{aligned}
t(\mathbf{n} \mid M) & =\frac{n_{1} y_{3}-n_{2} y_{3}}{Y_{23} Y_{13}}-\left\{\frac{\left(f_{23} Y_{13}+g_{23} Y_{12}\right)^{-1}\left(n_{1}\left(f_{23} x_{3}+g_{23} x_{2}\right)-n_{2}\left(f_{23} y_{3}+g_{23} y_{2}\right)\right)}{Y_{23}}\right\} \\
& -\left\{\frac{\left(f_{13} Y_{12}+g_{13} Y_{23}\right)^{-1}\left(n_{1}\left(f_{13} x_{1}+g_{13} x_{3}\right)-n_{2}\left(f_{13} y_{1}+g_{13} y_{3}\right)\right)}{Y_{13}}\right\}+1,
\end{aligned}
$$


where, $f_{12}, g_{12}, f_{13}, g_{13}, f_{23}$ and $g_{23} \in \mathbf{Z}$ satisfy $\operatorname{gcd}\left(f_{12} Y_{13}+g_{12} Y_{23}, Y_{12}\right)=1$ $\operatorname{gcd}\left(f_{13} Y_{12}+g_{13} Y_{23}, Y_{13}\right)=1$ and $g c d\left(f_{23} Y_{13}+g_{23} Y_{12}, Y_{23}\right)=1$, moreover, $\left(f_{12} Y_{13}+\right.$ $\left.g_{12} Y_{23}\right)^{-1}\left(f_{12} Y_{13}+g_{12} Y_{23}\right) \equiv 1 \bmod Y_{12},\left(f_{13} Y_{12}+g_{13} Y_{23}\right)^{-1}\left(f_{13} Y_{12}+g_{13} Y_{23}\right) \equiv$ $1 \bmod Y_{13},\left(f_{23} Y_{13}+g_{23} Y_{12}\right)^{-1}\left(f_{23} Y_{13}+g_{23} Y_{12}\right) \equiv 1 \bmod Y_{23}$.

Proof. We only prove the case where $\left(n_{1}, n_{2}\right)^{T} \in \bar{\Omega}_{1} \cap \mathbf{Z}^{2}$. Based on Theorem $3.2, p_{e, \Omega_{1}}(\mathbf{x})$, which is the polynomial part for $t(\cdot \mid M)$ on $\Omega_{1}$, is in the form of $p_{0, \Omega_{1}}(\mathbf{x})+p_{1, \Omega_{1}}(\mathbf{x})$. Here, when $\mathbf{x} \in \Omega_{1}, p_{0, \Omega_{1}}(\mathbf{x})=T(\mathbf{x} \mid M)$,

$p_{1, \Omega_{1}}(\mathbf{x})=-\left(\sum_{|v|=1} D^{v} p_{0, \Omega_{1}}(\mathbf{x})(-i) D^{v} \widehat{B}(0 \mid M)\right)$. By the explicit formulation for $T(\mathbf{x} \mid M)$, we have $p_{0, \Omega_{1}}(\mathbf{x})=\frac{y_{1} x-x_{1} y}{\left(x_{2} y_{1}-y_{2} x_{1}\right)\left(x_{1} y_{3}-y_{1} x_{3}\right)}$. After a brief calculation, we have $p_{1, \Omega}(\mathbf{x})=\frac{1}{2}\left(\frac{1}{Y_{13}}+\frac{1}{Y_{12}}\right)$. Hence, the polynomial part for $t(\mathbf{n} \mid M)$ is $\frac{n_{2} x_{1}-n_{1} y_{1}}{Y_{12} Y_{13}}+$ $\frac{1}{2}\left(\frac{1}{Y_{13}}+\frac{1}{Y_{12}}\right)$. According to Theorem 3.4, we only need to consider the sum

$$
\begin{aligned}
& \sum_{\theta \in A(M) \backslash e} \frac{1}{\left|\operatorname{det}\left(M_{\theta}\right)\right|} \prod_{w \in M \backslash M_{\theta}} \frac{\theta^{n}}{1-\theta^{-w}} 1_{\text {cone }\left(M_{\theta}\right)}\left(\Omega_{1}\right) \\
= & \frac{1}{Y_{12}} \sum_{\substack{\theta \in A(M) \backslash e \\
M_{\theta}=M_{12}}} \frac{\theta^{n}}{1-\theta^{-\left(x_{3}, y_{3}\right)}}+\frac{1}{Y_{13}} \sum_{\substack{\theta \in A(M) \backslash e \\
M_{\theta}=M_{13}}} \frac{\theta^{n}}{1-\theta^{-\left(x_{2}, y_{2}\right)}} .
\end{aligned}
$$

Recall $e^{\frac{2 \pi i}{k}}$ is denoted by $W_{k}$. As pointed out in [13], the elements in the set $\left\{\theta \mid \theta \in A(M), M_{\theta}=M_{12}\right\}$ have the form $\left(W_{Y_{12}}^{\alpha_{1}^{j}}, W_{Y_{12}}^{\alpha_{2}^{j}}\right)$, where $\left(\alpha_{1}^{j}, \alpha_{2}^{j}\right) \in \mathbf{Z}^{2}, 1 \leq$ $j \leq Y_{12}-1$.

Consider firstly

$$
\frac{1}{Y_{12}} \sum_{\substack{\theta \in A(M) \backslash e \\ M_{\theta}=M_{12}}} \frac{\theta^{\mathbf{n}}}{1-\theta^{-\left(x_{3}, y_{3}\right)}}=\frac{1}{Y_{12}} \sum_{j=1}^{Y_{12}-1} \frac{W_{Y_{12}}^{n_{1} \alpha_{1}^{j}+n_{2} \alpha_{2}^{j}}}{1-W_{Y_{12}}^{-\left(x_{3} \alpha_{1}^{j}+y_{3} \alpha_{2}^{j}\right)}} .
$$

We set $x_{3} \alpha_{1}^{j}+y_{3} \alpha_{2}^{j} \equiv k \bmod Y_{12}$. Since $M$ is a 1-prime matrix, $x_{3} \alpha_{1}^{j}+y_{3} \alpha_{2}^{j} \not \equiv$ $x_{3} \alpha_{1}^{m}+y_{3} \alpha_{2}^{m} \bmod Y_{12}$ when $j \neq m$. Hence, $k$ runs over $\left[1, Y_{12}-1\right] \cap \mathbf{Z}$.

For $\theta \in\left\{\theta \mid \theta \in A(M), M_{\theta}=M_{12}\right\}$, we have $\theta^{\left(x_{1}, y_{1}\right)}=\theta^{\left(x_{2}, y_{2}\right)}=1$. Hence,

$$
\begin{aligned}
& x_{1} \alpha_{1}^{j}+y_{1} \alpha_{2}^{j} \equiv 0 \bmod Y_{12}, \\
& x_{2} \alpha_{1}^{j}+y_{2} \alpha_{2}^{j} \equiv 0 \bmod Y_{12}, \\
& x_{3} \alpha_{1}^{j}+y_{3} \alpha_{2}^{j} \equiv k \bmod Y_{12} .
\end{aligned}
$$

By $x_{1}$ on both sides of (4.4), we have

$$
x_{1} x_{3} \alpha_{1}^{j}+x_{1} y_{3} \alpha_{2}^{j} \equiv x_{1} k \bmod Y_{12} .
$$

According to (4.2), we obtain

$$
x_{1} \alpha_{1}^{j} \equiv-y_{1} \alpha_{2}^{j} \bmod Y_{12} .
$$

Substituting (4.6) into (4.5), we have

$$
\alpha_{2}^{j} Y_{13} \equiv x_{1} k \bmod Y_{12} .
$$

By using similar method,

$$
\alpha_{2}^{j} Y_{23} \equiv x_{2} k \bmod Y_{12} .
$$


Since $M$ is a 1-prime matrix, there exist $f_{12}, g_{12} \in \mathbf{Z}$ such that $\operatorname{gcd}\left(f_{12} Y_{13}+\right.$ $\left.g_{12} Y_{23}, Y_{12}\right)=1$. Combining (4.7) and (4.8), we have

$$
\alpha_{2}^{j}\left(f_{12} Y_{13}+g_{12} Y_{23}\right) \equiv\left(f_{12} x_{1}+g_{12} x_{2}\right) k \bmod Y_{12} .
$$

Hence, $\alpha_{2}^{j} \equiv\left(f_{12} Y_{13}+g_{12} Y_{23}\right)^{-1}\left(f_{12} x_{1}+g_{12} x_{2}\right) k \bmod Y_{12}$.

Similarly, $\alpha_{1}^{j} \equiv-\left(f_{12} Y_{13}+g_{12} Y_{23}\right)^{-1}\left(f_{12} y_{1}+g_{12} y_{2}\right) k$ mod $Y_{12}$. Hence,(4.1) is reduced to

$$
\frac{1}{Y_{12}} \sum_{k=1}^{Y_{12}-1} \frac{W_{Y_{12}}^{\left(n_{2}\left(f_{12} x_{1}+g_{12} x_{2}\right)-n_{1}\left(f_{12} y_{1}+g_{12} y_{2}\right)\right)\left(f_{12} Y_{13}+g_{12} Y_{23}\right)^{-1} k}}{1-W_{Y_{12}}^{-k}} .
$$

According to discrete Fourier transforms,

$$
-\left\{\frac{t}{a}\right\}=\frac{1-a}{2 a}+\frac{1}{a} \sum_{k=1}^{a-1} \frac{W_{a}^{t k}}{1-W_{a}^{-k}},
$$

(4.9) can be reduced to

$$
\left\{\frac{\left(n_{2}\left(f_{12} x_{1}+g_{12} x_{2}\right)-n_{1}\left(f_{12} y_{1}+g_{12} y_{2}\right)\right)\left(f_{12} Y_{13}+g_{12} Y_{23}\right)^{-1}}{Y_{12}}\right\}+\frac{1}{2}-\frac{1}{2 Y_{12}} .
$$

Hence

$$
\begin{aligned}
& \frac{1}{Y_{12}} \sum_{\substack{\theta \in A(M) \\
M_{\theta}=Y_{12}}} \frac{\theta^{n}}{1-\theta^{-\left(x_{3}, y_{3}\right)}} \\
= & -\left\{\frac{\left(n_{2}\left(f_{12} x_{1}+g_{12} x_{2}\right)-n_{1}\left(f_{12} y_{1}+g_{12} y_{2}\right)\right)\left(f_{12} Y_{13}+g_{12} Y_{23}\right)^{-1}}{Y_{12}}\right\}+\frac{1}{2}-\frac{1}{2 Y_{12}} .
\end{aligned}
$$

By using similar method, we have

$$
\begin{aligned}
& \frac{1}{Y_{13}} \sum_{\substack{\theta \in A(M) \backslash e \\
M_{\theta}=Y_{13}}} \frac{\theta^{\mathbf{n}}}{1-\theta^{-\left(x_{2}, y_{2}\right)}} \\
= & -\left\{\frac{\left(n_{2}\left(f_{13} x_{1}+g_{13} x_{3}\right)-n_{1}\left(f_{13} y_{1}+g_{13} y_{3}\right)\right)\left(f_{13} Y_{12}+g_{13} Y_{23}\right)^{-1}}{Y_{13}}\right\}+\frac{1}{2}-\frac{1}{2 Y_{13}} .
\end{aligned}
$$

Hence, when $\left(n_{1}, n_{2}\right)^{T} \in v\left(\Omega_{1} \mid M\right) \cap \mathbf{Z}^{2}$,

$$
\begin{aligned}
& t(\mathbf{n} \mid M) \\
= & \frac{n_{2} x_{1}-n_{1} y_{1}}{Y_{12} Y_{13}}-\left\{\frac{\left(f_{12} Y_{13}+g_{12} Y_{23}\right)^{-1}\left(n_{2}\left(f_{12} x_{1}+g_{12} x_{2}\right)-n_{1}\left(f_{12} y_{1}+g_{12} y_{2}\right)\right)}{Y_{12}}\right\} \\
- & \left\{\frac{\left(f_{13} Y_{12}+g_{13} Y_{23}\right)^{-1}\left(n_{2}\left(f_{13} x_{1}+g_{13} x_{3}\right)-n_{1}\left(f_{13} y_{1}+g_{13} y_{3}\right)\right)}{Y_{13}}\right\}+1 .
\end{aligned}
$$

Note that $\bar{\Omega}_{1} \subset v\left(\Omega_{1} \mid M\right)$. Hence, when $\mathbf{n} \in \bar{\Omega}_{1} \cap \mathbf{Z}^{2}$, the theorem holds.

REMARK 4.4. If $f_{12}, g_{12}, f_{13}, g_{13}, f_{23}$ and $g_{23}$ satisfy $f_{12} Y_{23}+g_{12} Y_{13}=g c d\left(Y_{23}, Y_{13}\right)$, $f_{13} Y_{12}+g_{13} Y_{23}=\operatorname{gcd}\left(Y_{12}, Y_{23}\right), f_{23} Y_{13}+g_{23} Y_{12}=\operatorname{gcd}\left(Y_{13}, Y_{12}\right)$, then $\operatorname{gcd}\left(f_{12} Y_{13}+\right.$ $\left.g_{12} Y_{23}, Y_{12}\right)=1, \operatorname{gcd}\left(f_{13} Y_{12}+g_{13} Y_{23}, Y_{13}\right)=1$ and $\operatorname{gcd}\left(f_{23} Y_{13}+g_{23} Y_{12}, Y_{23}\right)=1$. Hence,one can determine $f_{12}, g_{12}, f_{13}, g_{13}, f_{23}$ and $g_{23}$ by Euclidean algorithm. But in some special cases, such as $Y_{12}, Y_{13}$ and $Y_{23}$ are pairwise relative prime, there exists a simpler method for obtaining them. 
Corollary 4.5. Suppose $Y_{12}, Y_{13}$ and $Y_{23}$ are pairwise relative prime. When $\mathbf{n}=\left(n_{1}, n_{2}\right)^{T} \in \bar{\Omega}_{1} \cap \mathbf{Z}^{2}$,

$$
t(\mathbf{n} \mid M)=\frac{n_{2} x_{1}-n_{1} y_{1}}{Y_{12} Y_{13}}-\left\{\frac{Y_{13}^{-1}\left(n_{2} x_{1}-n_{1} y_{1}\right)}{Y_{12}}\right\}-\left\{\frac{Y_{12}^{-1}\left(n_{2} x_{1}-n_{1} y_{1}\right)}{Y_{13}}\right\}+1,
$$

where $Y_{13}^{-1} Y_{13} \equiv 1 \bmod Y_{12}$ and $Y_{12}^{-1} Y_{12} \equiv 1 \bmod Y_{13}$. When $\mathbf{n}=\left(n_{1}, n_{2}\right)^{T} \in$ $\bar{\Omega}_{2} \cap \mathbf{Z}^{2}$

$$
t(\mathbf{n} \mid M)=\frac{n_{1} y_{3}-n_{2} x_{3}}{Y_{23} Y_{13}}-\left\{\frac{Y_{13}^{-1}\left(n_{1} x_{3}-n_{2} y_{3}\right)}{Y_{23}}\right\}-\left\{\frac{Y_{23}^{-1}\left(n_{1} x_{3}-n_{2} y_{3}\right)}{Y_{13}}\right\}+1,
$$

where $Y_{13}^{-1} Y_{13} \equiv 1 \bmod Y_{23}$ and $Y_{23}^{-1} Y_{23} \equiv 1 \bmod Y_{13}$.

Proof. We firstly consider the case where $\mathbf{n} \in \bar{\Omega}_{1} \cap \mathbf{Z}^{2}$. Since $\operatorname{gcd}\left(Y_{12}, Y_{13}\right)=1$, $M$ is a 1 -prime matrix. In Theorem 4.3 , we may set $f_{12}=1, g_{12}=0, f_{13}=1$, and $g_{13}=0$. Hence, When $\mathbf{n}=\left(n_{1}, n_{2}\right)^{T} \in \bar{\Omega}_{1} \cap \mathbf{Z}^{2}$,

$$
t(\mathbf{n} \mid M)=\frac{n_{2} x_{1}-n_{1} y_{1}}{Y_{12} Y_{13}}-\left\{\frac{Y_{13}^{-1}\left(n_{2} x_{1}-n_{1} y_{1}\right)}{Y_{12}}\right\}-\left\{\frac{Y_{12}^{-1}\left(n_{2} x_{1}-n_{1} y_{1}\right)}{Y_{13}}\right\}+1 .
$$

Using similar method, when $\mathbf{n}=\left(n_{1}, n_{2}\right)^{T} \in \bar{\Omega}_{2} \cap \mathbf{Z}^{2}$,

$$
t(\mathbf{n} \mid M)=\frac{n_{1} y_{3}-n_{2} x_{3}}{Y_{23} Y_{13}}-\left\{\frac{Y_{13}^{-1}\left(n_{1} x_{3}-n_{2} y_{3}\right)}{Y_{23}}\right\}-\left\{\frac{Y_{23}^{-1}\left(n_{1} x_{3}-n_{2} y_{3}\right)}{Y_{13}}\right\}+1 \text {. }
$$

REMARK 4.6. An interesting observation is that the formulation presented in Corollary 6.2 is remarkably similar with Popoviciu's formulation.

We now turn to consider the special case where $\frac{y_{1}}{x_{1}}=\frac{y_{2}}{x_{2}}$. Without loss of generality, we suppose $M=\left(\begin{array}{lll}k x_{1} & l x_{1} & x_{3} \\ k y_{1} & l y_{1} & y_{3}\end{array}\right)$, where $k, l \in \mathbf{Z}$. In this case, there exists only one fundamental $M$-cone, which is denoted as $\Omega$. Moreover, since $M$ is a 1 -prime matrix, we have $\operatorname{gcd}(k, l)=1, x_{1} y_{3}-y_{1} x_{3}=1$. Then we have

TheOREM 4.7. Suppose $\frac{y_{1}}{x_{1}}<\frac{y_{3}}{x_{3}}$. When $M=\left(\begin{array}{lll}k x_{1} & l x_{1} & x_{3} \\ k y_{1} & l y_{1} & y_{3}\end{array}\right), t(\mathbf{n} \mid M)=$ $\frac{x_{3} n_{2}-y_{3} n_{1}}{k l}-\left\{\frac{l^{-1}}{k}\left(n_{1} y_{3}-n_{2} x_{3}\right)\right\}-\left\{\frac{k^{-1}}{l}\left(n_{1} y_{3}-n_{2} x_{3}\right)\right\}+1$, where $\mathbf{n}=\left(n_{1}, n_{2}\right)^{T} \in$ $\bar{\Omega} \cap \mathbf{Z}^{2}, k^{-1} k \equiv 1 \bmod l, l^{-1} l \equiv 1 \bmod k$.

ProOF. By using the recurrence formulation for $T(\mathbf{x} \mid M)$, we have $T(\mathbf{x} \mid M)=$ $\frac{x_{3} y-y_{3} x}{k l}$. Hence, the polynomial part of $t(\cdot \mid M)$ is $\frac{x_{3} y-y_{3} x}{k l}+\frac{1}{2}\left(\frac{1}{k}+\frac{1}{l}\right)$. We now only need to consider the sums

$$
\frac{1}{k} \sum_{\theta: M_{\theta}=Y_{k}} \frac{\theta^{n}}{1-\theta^{-\left(l x_{1}, l y_{1}\right)}}, \frac{1}{l} \sum_{\theta: M_{\theta}=Y_{l}} \frac{\theta^{n}}{1-\theta^{-\left(k x_{1}, k y_{1}\right)}} .
$$

By the similar method with the one presented in the proof of Theorem 4.3, we have $\frac{1}{k} \sum_{\substack{\theta \in A(M) \backslash e \\ M_{\theta}=Y_{k}}} \frac{\theta^{n}}{1-\theta^{-\left(l x_{1}, l y_{1}\right)}}=-\left\{l^{-1} \frac{n_{1} y_{3}-n_{2} x_{3}}{k}\right\}+\frac{1}{2}-\frac{1}{2 k}, \frac{1}{l} \sum_{\substack{\theta \in A(M) \\ M_{\theta}=Y_{l}}} \frac{\theta^{n}}{1-\theta^{-\left(k x_{1}, k y_{1}\right)}}=$ $-\left\{k^{-1} \frac{n_{1} y_{3}-n_{2} x_{3}}{k}\right\}+\frac{1}{2}-\frac{1}{2 l}$. Note that $\bar{\Omega} \subset v(\Omega \mid M)$. According to Theorem 3.4, 
when $\mathbf{n}=\left(n_{1}, n_{2}\right)^{T} \in \bar{\Omega}$,

$$
\begin{aligned}
& t(\mathbf{n} \mid M) \\
= & \frac{x_{3} y-y_{3} x}{k l}+\frac{1}{2}\left(\frac{1}{k}+\frac{1}{l}\right)+\frac{1}{k} \sum_{\theta: M_{\theta}=Y_{k}} \frac{\theta^{n}}{1-\theta^{-\left(l x_{1}, l y_{1}\right)}}+\frac{1}{l} \sum_{\theta: M_{\theta}=Y_{l}} \frac{\theta^{n}}{1-\theta^{-\left(k x_{1}, k y_{1}\right)}} \\
= & \frac{x_{3} n_{2}-y_{3} n_{1}}{k l}-\left\{\frac{l^{-1}}{k}\left(n_{1} y_{3}-n_{2} x_{3}\right)\right\}-\left\{\frac{k^{-1}}{l}\left(n_{1} y_{3}-n_{2} x_{3}\right)\right\}+1 .
\end{aligned}
$$

REMARK 4.8. When the matrix $M$ is of the form $\left(\begin{array}{lll}x_{1} & k x_{2} & l x_{2} \\ y_{1} & k y_{2} & l y_{2}\end{array}\right)$, a similar result can be obtained using the same method with the one presented in Theorem 4.7 .

\section{Linear Diophantine problem of Frobenius}

Consider the linear Diophantine equation

$$
x_{1} a_{1}+\cdots x_{n} a_{n}=N
$$

where, $a_{i} \in \mathbf{Z}_{+}, \operatorname{gcd}\left(a_{1}, \cdots, a_{n}\right)=1$.

It is well known that for all sufficiently large $N$ the equation has solutions. The Frobenius problems asks us to find the largest integer for which no solution exists. We call the largest integer the Frobenius number and denote it by $f\left(a_{1}, \cdots, a_{n}\right)$. For $n=2$ the largest $N$ for which no solution exists can be explicitly written as $a_{1} a_{2}-a_{1}-a_{2}$, i.e. $f\left(a_{1}, a_{2}\right)=a_{1} a_{2}-a_{1}-a_{2}$. But,to our knowledge, no such formula exists for $n \geq 3$.

As pointed out in [34], when $\operatorname{gcd}\{|Y|: Y \in \mathcal{B}(M)\}=1$, for all sufficiently large $N$ the linear Diophantine equations $M x=N \mathbf{n}$ has solution, where $\mathbf{n} \in \operatorname{cone}(M)$. Naturally, we hope to find the largest integer $N$ for which no solution exist, which is denoted as $f(M, \mathbf{n})$. In particular, we are interested in the linear Diophantine equations $M_{0} x=N \mathbf{n}$, where $M_{0}=\left(\begin{array}{lll}x_{1} & x_{2} & x_{3} \\ y_{1} & y_{2} & y_{3}\end{array}\right) \in \mathbf{Z}^{2 \times 3}, \mathbf{n} \in$ cone $\left(M_{0}\right)$. In fact, the generalized Frobenius number $f\left(M_{0}, \mathbf{n}\right)$ is a generalization of $f\left(a_{1}, a_{2}\right)$.

Recall $M_{i j}=\left(\begin{array}{ll}x_{i} & x_{j} \\ y_{i} & y_{j}\end{array}\right)$ and $Y_{i j}=\operatorname{det}\left(M_{i j}\right)$. In the following theorem, we shall present an upper boundary for $f\left(M_{0}, \mathbf{n}\right)$.

TheOREM 5.1. Suppose $Y_{12}, Y_{13}$ and $Y_{23}$ are pairwise relative prime. For $\mathbf{n} \in$ $\bar{\Omega}_{1} \cap \mathbf{Z}^{2}, f\left(M_{0}, \mathbf{n}\right)<\frac{Y_{12} Y_{13}-Y_{12}-Y_{13}+1}{n_{2} x_{1}-n_{1} y_{1}}$. For $\mathbf{n} \in \bar{\Omega}_{2} \cap \mathbf{Z}^{2}, f\left(M_{0}, \mathbf{n}\right)<\frac{Y_{23} Y_{13}-Y_{23}-Y_{13}+1}{n_{1} y_{3}-n_{2} x_{3}}$.

Proof. We only prove the case where $\mathbf{n} \in \bar{\Omega}_{1} \cap \mathbf{Z}^{2}$. Note $t(N \mathbf{n} \mid M)=\frac{N\left(n_{2} x_{1}-n_{1} y_{1}\right)}{Y_{12} Y_{13}}-$ $\left\{\frac{\left(Y_{13}\right)^{-1}\left(N\left(n_{2} x_{1}-n_{1} y_{1}\right)\right)}{Y_{12}}\right\}-\left\{\frac{\left(Y_{12}\right)^{-1}\left(N\left(n_{2} x_{1}-n_{1} y_{1}\right)\right)}{Y_{13}}\right\}+1=t\left(N\left(n_{2} x_{1}-n_{1} y_{1}\right) \mid\left(Y_{12}, Y_{13}\right)\right)$. When $N\left(n_{2} x_{1}-n_{1} y_{1}\right) \geq Y_{12} Y_{13}-Y_{12}-Y_{13}+1, t\left(N\left(n_{2} x_{1}-n_{1} y_{1}\right) \mid Y_{12}, Y_{13}\right)=$ $t(N \mathbf{n} \mid M)>0$. Hence, when $N \geq \frac{Y_{12} Y_{13}-Y_{12}-Y_{13}+1}{\left(n_{2} x_{1}-n_{1} y_{1}\right)}, t(N \mathbf{n} \mid M)>0$. So, $f(M, \mathbf{n})<$ $\frac{Y_{12} Y_{13}-Y_{12}-Y_{13}+1}{n_{2} x_{1}-n_{1} y_{1}}$.

Remark 5.2. Theorem 5.1 only gives an upper boundary for $f\left(M_{0}, \mathbf{n}\right)$. According to the proof of Theorem 5.1, giving the exact value of $f\left(M_{0}, \mathbf{n}\right)$ is equivalent for any given $b_{0} \in \mathbf{Z}$ determining the largest integer $N$ for which the Diophantine equation $x_{1} a_{1}+x_{2} a_{2}=N b_{0}$ no solution exist. 


\section{Two-dimension vector partition functions}

We now turn to the general case. We let $M=\left(\begin{array}{llll}x_{1} & x_{2} & \cdots & x_{n} \\ y_{1} & y_{2} & \cdots & y_{n}\end{array}\right)$ be a $2 \times n$ integer matrix and $\frac{y_{i-1}}{x_{i-1}}<\frac{y_{i}}{x_{i}}, i=2, \cdots, n$.

For the matrix $M$, there exist $n-1$ fundamental $M$-cones. Denote them as $\Omega_{i}:=\left\{(x, y)^{T} \mid(x, y)^{T} \in \operatorname{cone}(M), \frac{y_{i}}{x_{i}}<\frac{y}{x}<\frac{y_{i+1}}{x_{i+1}}\right\}, i=1, \cdots, n-1$ respectively. In this section, we shall discuss the explicit formulation for $t(\mathbf{b} \mid M)$. First, we present an explicit formulation for $T(\mathbf{x} \mid M)$.

Theorem 6.1. For $\mathbf{x}=(x, y)^{T} \in \mathbf{R}^{2}$,

$$
T(\mathbf{x} \mid M)=\frac{1}{(n-2) !} \sum_{i=1}^{n} \frac{\left(y_{i} x-x_{i} y\right)_{+}^{n-2}}{\prod_{j \neq i}\left(y_{i} x_{j}-y_{j} x_{i}\right)}
$$

where, $\left(y_{i} x-x_{i} y\right)_{+}= \begin{cases}y_{i} x-x_{i} y, & y_{i} x-x_{i} y \geq 0, \\ 0, & \text { otherwise. }\end{cases}$

Proof. According to the definition of $\left(y_{i} x-x_{i} y\right)_{+}$we only need to prove that when $\mathbf{x} \in \Omega_{k}, T(\mathbf{x} \mid M)=\frac{1}{(n-2) !} \sum_{i=k+1}^{n} \frac{\left(y_{i} x-x_{i} y\right)^{n-2}}{\prod_{j \neq i}\left(y_{i} x_{j}-y_{j} x_{i}\right)}$.

We argue by induction on $n$. Initially, when $n=2,3$ the theorem certainly holds. In the inductive step, we assume that when $n=n_{0}$ the theorem holds and we consider the case when $n=n_{0}+1$.

According to the definition of $\left(y_{i} x-x_{i} y\right)_{+}$we only need to prove that for $\mathbf{x} \in \Omega_{k}, T(\mathbf{x} \mid M)=\frac{1}{\left(n_{0}-1\right) !} \sum_{i=k+1}^{n_{0}+1} \frac{\left(y_{i} x-x_{i} y\right)^{n_{0}-1}}{\prod_{j \neq i}\left(y_{i} x_{j}-y_{j} x_{i}\right)}$, where $M$ is a $2 \times\left(n_{0}+1\right)$ matrix.

After a brief calculation, it is easy for obtaining $\mathbf{x}=\frac{x y_{k+1}-x_{k+1} y}{y_{k+1} x_{k}-y_{k} x_{k+1}}\left(x_{k}, y_{k}\right)^{T}+$ $\frac{x y_{k}-x_{k} y}{y_{k+1} x_{k}-y_{k} x_{k+1}}\left(x_{k+1}, y_{k+1}\right)^{T}$. Based on the recurrence formulation of $T(\cdot \mid M)$, we have

$$
\begin{array}{r}
T(\mathbf{x} \mid M)=\frac{1}{n_{0}-1}\left(\frac{x y_{k+1}-x_{k+1} y}{y_{k+1} x_{k}-y_{k} x_{k+1}} T\left(\mathbf{x} \mid M \backslash\left(x_{k}, y_{k}\right)^{T}\right)+\right. \\
\left.\frac{x y_{k}-x_{k} y}{y_{k+1} x_{k}-y_{k} x_{k+1}} T\left(\mathbf{x} \mid M \backslash\left(x_{k+1}, y_{k+1}\right)^{T}\right)\right) .
\end{array}
$$


By the inductive hypothesis, $T\left(\mathbf{x} \mid M \backslash\left(x_{k}, y_{k}\right)^{T}\right)=\frac{1}{\left(n_{0}-2\right) !} \sum_{i=k+1}^{n_{0}+1} \frac{\left(y_{i} x-x_{i} y\right)^{n_{0}-2}}{\prod_{j \neq i, j \neq k}\left(y_{i} x_{j}-y_{j} x_{i}\right)}$, $T\left(\mathbf{x} \mid M \backslash\left(x_{k+1}, y_{k+1}\right)^{T}\right)=\frac{1}{\left(n_{0}-2\right) !} \sum_{i=k+2}^{n_{0}+1} \frac{\left(y_{i} x-x_{i} y\right)^{n_{0}-2}}{\prod_{j \neq i}\left(y_{i} x_{j}-y_{j} x_{i}\right)}$. Then we obtain

$$
\begin{aligned}
& T(\mathbf{x} \mid M)=\frac{1}{\left(n_{0}-1\right) !}\left(\frac{x y_{k+1}-x_{k+1} y}{y_{k+1} x_{k}-y_{k} x_{k+1}} \sum_{i=k+1}^{n_{0}+1} \frac{\left(y_{i} x-x_{i} y\right)^{n_{0}-2}\left(x_{k} y_{i}-y_{k} x_{i}\right)}{\prod_{j \neq i}\left(y_{i} x_{j}-y_{j} x_{i}\right)}\right. \\
+ & \left.\frac{x y_{k}-x_{k} y}{y_{k+1} x_{k}-y_{k} x_{k+1}} \sum_{i=k+2}^{n_{0}+1} \frac{\left(y_{i} x-x_{i} y\right)^{n_{0}-2}\left(x_{k+1} y_{i}-y_{k+1} x_{i}\right)}{\prod_{j \neq i}\left(y_{i} x_{j}-y_{j} x_{i}\right)}\right) \\
= & \frac{1}{\left(n_{0}-1\right) !}\left(\frac{\left(y_{k+1} x-x_{k+1} y\right)^{n_{0}-1}}{\prod_{j \neq k+1}\left(y_{k+1} x_{j}-y_{j} x_{k+1}\right)}+\frac{1}{y_{k+1} x_{k}-y_{k} x_{k+1}} \sum_{i=k+2}^{n_{0}+1}\left(y_{i} x-x_{i} y\right)^{n_{0}-2}\right. \\
& \left.\left(\frac{\left(x y_{k+1}-x_{k+1} y\right)\left(x_{k} y_{i}-y_{k} x_{i}\right)-\left(x y_{k}-x_{k} y\right)\left(x_{k+1} y_{i}-y_{k+1} x_{i}\right)}{\prod_{j \neq i}\left(y_{i} x_{j}-y_{j} x_{i}\right)}\right)\right) \\
= & \frac{1}{\left(n_{0}-1\right) !} \sum_{i=k+1}^{n_{0}+1} \frac{\left(y_{i} x-x_{i} y\right)^{n_{0}-1}}{\prod_{j \neq i}\left(y_{i} x_{j}-y_{j} x_{i}\right)} .
\end{aligned}
$$

Thus, when $n=n_{0}+1$ the theorem holds also, which completes the inductive step and the proof.

The following statements follow from Theorem 6.1.

Corollary 6.2 .

$$
D^{v_{1}, v_{2}} T(\mathbf{x} \mid M)=\frac{1}{\left(n-2-v_{1}-v_{2}\right) !} \sum_{i=1}^{n} \frac{\left(y_{i} x-x_{i} y\right)_{+}^{n-2-v_{1}-v_{2}}}{\prod_{j \neq i}\left(y_{i} x_{j}-y_{j} x_{i}\right)} y_{i}^{v_{1}}\left(-x_{i}\right)^{v_{2}} .
$$

We now turn to the non-polynomial part for $t(\cdot \mid M)$. We firstly recall the definition of a Fourier-Dedekind sum(see [1] ), which is defined as $\sigma_{t}(C ; n)=\frac{1}{n} \sum_{\lambda^{n}=1 \neq \lambda} \frac{\lambda^{t}}{\prod_{c \in C}\left(\lambda^{c}-1\right)}$, where $C$ is an integer multiset and $n$ is an integer. To simplify the non-polynomial part for $t(\cdot \mid M)$, we naturally arrived at the sums

$$
\frac{1}{Y_{i j}} \sum_{\theta^{M_{i j}}=1, \theta \neq e} \theta^{n} \prod_{\omega \in M \backslash M_{i j}} \frac{1}{1-\theta^{-\omega}},
$$

which is considered as a generalized Fourier-Dedekind sum. Here, $\theta^{M_{i j}}=1$ means $\theta^{m}=1$ for any $m \in M_{i j}$. In fact, it is a non-trivial problem for computing all complex vectors satisfying $\theta^{M_{i j}}=1$. In the following Lemma, we shows the generalized Fourier-Dedekind sums (6.1) can be converted into the 1-dimensional FourierDedekind sums.

LEMMA 6.3. When $M$ is a 1-prime matrix, for any given integer $m, 1 \leq m \leq$ $n, m \neq i, j$,

$$
\frac{1}{Y_{i j}} \sum_{\substack{\theta^{M_{i j}=1} \\ \theta \neq e}} \theta^{n} \prod_{\omega \in M \backslash M_{i j}} \frac{1}{1-\theta^{-\omega}}=\sigma_{t_{i j}}\left(C_{i j} ; Y_{i j}\right)
$$


Here, $C_{i j}=\cup_{1 \leq h \leq n, h \neq i, h \neq j}\left\{\left(f Y_{i m}+g Y_{j m}\right)^{-1}\left(-\left(f y_{i}+g y_{j}\right) x_{h}+\left(f x_{i}+g x_{j}\right) y_{h}\right)\right\}, t_{i j}=$ $\left(f Y_{i m}+g Y_{j m}\right)^{-1}\left(-\left(f y_{i}+g y_{j}\right) n_{1}+\left(f x_{i}+g x_{j}\right) n_{2}\right)+\sum_{c \in C_{i j}} c$, where $f, g \in \mathbf{Z}$ satisfy $g c d\left(f Y_{i m}+g Y_{j m}, Y_{i j}\right)=1,\left(f Y_{i m}+g Y_{j m}\right)^{-1}\left(f Y_{i m}+g Y_{j m}\right) \equiv 1 \bmod Y_{i j}$.

Proof. As pointed out in [13], the elements in the set $\left\{\theta \mid \theta \in A(M), M_{\theta}=\right.$ $\left.M_{i j}\right\}$ have the form $\left(W_{Y_{i j}}^{\alpha_{1}^{l}}, W_{Y_{12}}^{\alpha_{2}^{l}}\right)$, where $\left(\alpha_{1}^{l}, \alpha_{2}^{l}\right) \in \mathbf{Z}^{2}, 1 \leq l \leq Y_{i j}$.

Hence,

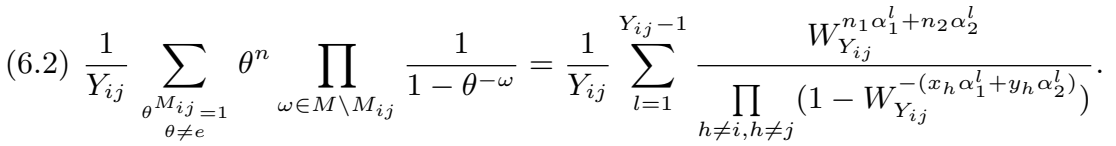

Noting $m \neq i, m \neq j$, we set $x_{m} \alpha_{1}^{l}+y_{m} \alpha_{2}^{l} \equiv k \bmod Y_{i j}$. Since $M$ is a 1-prime matrix, $k$ runs over $\left[1, Y_{i j}-1\right] \cap \mathbf{Z}$. Using the similar method with the one in the proof of Theorem 4.3, we have

$$
\begin{aligned}
\alpha_{1}^{l} & \equiv-\left(f_{i j} Y_{i m}+g_{i j} Y_{j m}\right)^{-1}\left(f_{i j} y_{i}+g_{i j} y_{j}\right) k \bmod Y_{i j}, \\
\alpha_{2}^{l} & \equiv\left(f_{i j} Y_{i m}+g_{i j} Y_{j m}\right)^{-1}\left(f_{i j} x_{i}+g_{i j} x_{j}\right) k \bmod Y_{i j} .
\end{aligned}
$$

Hence, (6.2) is reduced to

$$
\begin{aligned}
& \frac{1}{Y_{i j}} \sum_{k=1}^{Y_{i j}-1} \frac{W_{Y_{i j}}^{\left(n_{2}\left(f_{i j} x_{i}+g_{i j} x_{j}\right)-n_{1}\left(f_{i j} y_{i}+g_{12} y_{j}\right)\right)\left(f_{i j} Y_{i m}+g_{i j} Y_{j m}\right)^{-1} k}}{\prod_{h \neq i, h \neq j}\left(1-W_{Y_{i j}}^{-\left(f_{i j} Y_{i m}+g_{i j} Y_{j m}\right)^{-1}\left(-x_{h}\left(f_{i j} y_{i}+g_{i j} y_{j}\right)+y_{h}\left(f_{i j} x_{i}+g_{i j} x_{j}\right)\right) k}\right)} \\
= & \sigma_{t_{i j}}\left(C_{i j} ; Y_{i j}\right) .
\end{aligned}
$$

Remark 6.4. When $\left|\operatorname{det}\left(M_{i j}\right)\right|=1$, the terms in $\sigma_{t_{i j}}\left(C_{i j}: Y_{i j}\right)$ disappear,since $\left\{\theta: \theta^{M_{i j}}=1\right\}=\{e\}$.

Combining Theorem 3.2, Theorem 3.4, Theorem 6.1 and Lemma 6.3, we can present a simplified formulation for $t(\cdot \mid M)$.

Theorem 6.5. Suppose $M=\left(\begin{array}{llll}x_{1} & x_{2} & \cdots & x_{n} \\ y_{1} & y_{2} & \cdots & y_{n}\end{array}\right)$ is a $2 \times n$ integer 1-prime matrix and $\frac{y_{i}}{x_{i}}<\frac{y_{i+1}}{x_{i+1}}$. When $\mathbf{n}=\left(n_{1}, n_{2}\right)^{T} \in \Omega_{k} \cap \mathbf{Z}^{2}$,

$$
t(\mathbf{n} \mid M)=p_{e, \Omega_{k}}(\mathbf{n})+\sum_{(i, j) \in\{(i, j): i \leq k<j\}} \sigma_{t_{i j}}\left(C_{i j} ; Y_{i j}\right),
$$

where, $p_{e, \Omega_{k}}(\mathbf{x})=\sum_{j=0}^{n-2} p_{j, \Omega_{k}}(\mathbf{x}), p_{0, \Omega_{k}}(\mathbf{x})=\frac{1}{n-2} \sum_{l=k+1}^{n} \frac{\left(y_{l} x-x_{l} y\right)^{n-2}}{\prod_{j \neq l}\left(y_{l} x_{j}-y_{j} x_{l}\right)}, p_{j, \Omega_{k}}(\mathbf{x})=$ $-\sum_{l=0}^{j-1}\left(\sum_{|v|=j-l} D^{v} p_{l, \Omega_{k}}(\mathbf{x})(-i)^{|v|} \frac{D^{v} \widehat{B}(0 \mid M)}{v !}\right), t_{i j}$ and $C_{i j}$ are defined in Lemma

Proof. Based on Theorem 3.4, when $\mathbf{n} \in \Omega_{k} \cap \mathbf{Z}^{2}$,

$$
t(\mathbf{n} \mid M)=p_{e, \Omega_{k}}(\mathbf{n})+\sum_{\theta \in A(M) \backslash e} \theta^{\mathbf{n}} \frac{1}{\left|\operatorname{det}\left(M_{\theta}\right)\right|} \prod_{w \in M \backslash M_{\theta}} \frac{1}{1-\theta^{-w}} 1_{\operatorname{cone}\left(M_{\theta}\right)}\left(\Omega_{k}\right),
$$


where, the $p_{e, \Omega_{k}}$ can be determined easily. Since $M$ is a 1 -prime matrix,

$$
\begin{aligned}
& \sum_{\theta \in A(M) \backslash e} \theta^{\mathbf{n}} \frac{1}{\left|\operatorname{det}\left(M_{\theta}\right)\right|} \prod_{w \in M \backslash M_{\theta}} \frac{1}{1-\theta^{-w}} 1_{\operatorname{cone}\left(M_{\theta}\right)}\left(\Omega_{k}\right) \\
= & \sum_{i<j} \frac{1}{Y_{i j}} \sum_{\substack{\theta^{M_{i j}} \\
\theta \neq e}} \theta^{\mathbf{n}} \prod_{\omega \in M \backslash M_{i j}} \frac{1}{1-\theta^{-\omega}} 1_{\operatorname{cone}\left(M_{i j}\right)}\left(\Omega_{k}\right) .
\end{aligned}
$$

Based on Lemma 6.3, the above sum becomes as follows:

$$
\sum_{i<j} \sigma_{t_{i j}}\left(C_{i j}: Y_{i j}\right) 1_{\text {cone }\left(M_{i j}\right)}\left(\Omega_{k}\right) .
$$

Since when $k \geq j$ or $k<i$, cone $\left(M_{i j}\right) \cap \Omega_{k}=\emptyset$. Hence, (6.3) is converted into

$$
\sum_{(i, j) \in\{(i, j): i \leq k<j\}} \sigma_{t_{i j}}\left(C_{i j}: Y_{i j}\right) .
$$

The theorem holds.

Using Theorem 6.5, we shall present an explicit formulation for an actual example, which is the same with the one presented in [3]. By using Theorem 6.5, it is indeed easier for obtaining the explicit formulation for the actual vector partition function.

ExAmple 6.6. Let $A=\left(\begin{array}{llll}1 & 2 & 1 & 0 \\ 0 & 1 & 1 & 1\end{array}\right)$. We denote by $A_{i j}$ the square matrix containing the $i$ th and the $j$ th columns in $A$.

For the matrix $A$, there exist three fundamental cones, which are denoted as $\Omega_{1}, \Omega_{2}$ and $\Omega_{3}$ respectively. We shall discuss the explicit formulation for $t(\mathbf{n} \mid A)$. After a brief calculation, we have

$$
T(\mathbf{x} \mid A)= \begin{cases}\frac{y^{2}}{2}, & \mathbf{x} \in \Omega_{1}, \\ \frac{1}{4}\left(-x^{2}+4 x y-2 y^{2}\right), & \mathbf{x} \in \Omega_{2}, \\ \frac{x^{2}}{4}, & \mathbf{x} \in \Omega_{3} .\end{cases}
$$

Hence, $p_{0, \Omega_{1}}=\frac{y^{2}}{2}$. According to Theorem $6.1, p_{1, \Omega_{1}}=3 / 2 y$ and $p_{2, \Omega_{1}}=1$ respectively. Since for any $1<j \leq 3,\left|\operatorname{det}\left(A_{1 j}\right)\right|=1$, accord to Remark 6.4, the terms in the Fourier-Dedekind sum shall not appear when $\mathbf{n} \in \Omega_{1} \cap \mathbf{Z}^{2}$. Based on Theorem 6.1, we have when $\mathbf{n} \in \Omega_{1} \cap \mathbf{Z}^{2}, t(\mathbf{n} \mid \mathbf{A})=\frac{n_{2}^{2}}{2}+\frac{3 n_{2}}{2}+1$.

Similarly, $p_{0, \Omega_{2}}=\frac{1}{4}\left(-x^{2}+4 x y-2 y^{2}\right), p_{1, \Omega_{2}}=\frac{x+y}{2}, p_{2, \Omega_{2}}=\frac{7}{8}$. Based on Lemma 6.3, the non-polynomial part is $\frac{1}{Y_{23}} \sum_{\theta^{A_{23}}=1, \theta \neq e} \theta^{n} \prod_{\omega \in A \backslash A_{i j}} \frac{1}{1-\theta^{-\omega}}=(-1)^{n_{1}}$. Hence, when $\mathbf{n} \in \Omega_{2} \cap \mathbf{Z}^{2}, t(\mathbf{n} \mid A)=n_{1} n_{2}-\frac{n_{1}^{2}}{4}-\frac{n_{2}^{2}}{2}+\frac{n_{1}+n_{2}}{2}+\frac{7}{8}+\frac{(-1)^{n_{1}}}{8}$. $\frac{7}{8}$.

Using the same method with the above, we obtain $p_{0, \Omega_{3}}=\frac{x^{2}}{4}, p_{1, \Omega_{3}}=x, p_{2, \Omega_{3}}=$

$$
\text { Hence, } t(\mathbf{n} \mid A)= \begin{cases}\frac{n_{2}^{2}}{2}+\frac{3 n_{2}}{2}+1, & \mathbf{n} \in \Omega_{1} \cap \mathbf{Z}^{2} \\ n_{1} n_{2}-\frac{n_{1}^{2}}{4}-\frac{n_{2}^{2}}{2}+\frac{n_{1}+n_{2}}{2}+\frac{7}{8}+\frac{(-1)^{n_{1}}}{8}, & \mathbf{n} \in \Omega_{2} \cap \mathbf{Z}^{2} \\ \frac{n_{1}^{2}}{4}+n_{1}+\frac{7}{8}+\frac{(-1)^{n_{1}}}{8}, & \mathbf{n} \in \Omega_{3} \cap \mathbf{Z}^{2} .\end{cases}
$$


REMARK 6.7. The explicit formulation presented in Theorem 6.5 contains $D^{v} \widehat{B}(0 \mid M)$. Note

$$
\widehat{B}(\zeta \mid M)=\prod_{j=1}^{n} \frac{1-\exp \left(-i \zeta^{T} m_{j}\right)}{i \zeta^{T} m_{j}}, \zeta \in \mathbf{C}^{s} .
$$

The following assertion is obvious:

$$
D^{v_{1}, v_{2}} \widehat{B}(0 \mid M)=(-i)^{v_{1}+v_{2}} \sum_{k_{1}+\cdots+k_{n}=v_{1}} \sum_{l_{1}+\cdots+l_{n}=v_{2}} \frac{v_{1} !}{k_{1} ! \cdots k_{n} !} \frac{v_{2} !}{l_{1} ! \cdots l_{n} !} \prod_{j=1}^{n} \frac{x_{j}^{k_{j}} y_{j}^{l_{j}}}{k_{j}+l_{j}+1} .
$$

REMARK 6.8. In Theorem 6.5, when the case of $\frac{y_{i}}{x_{i}}=\frac{y_{j}}{x_{j}}$ happens, the explicit formulation for $T(\mathbf{x} \mid M)$ can be obtained by taking the limit. Using similar method with the one in the proof of Theorem 4.7, an explicit formulation for $t(\mathbf{n} \mid M)$ can be given also.

REMARK 6.9. To simplify any-dimensional vector partition functions, we have to give an explicit formulation for multivariate truncated power functions $T(\mathbf{x} \mid M)$ and compute the chamber complex consisting of the fundamental $M$-cones, which are indeed challenging problems.

\section{References}

[1] M. Beck, R. Diaz and S. Robins, The Frobenius Problem, Rational Polytopes, and Fourier-Dedekind Sums, J. Number Theory.,(96) (2002), 1-21.

[2] M. Beck, and S. Robins, Computing the continuous discretely integer point enumeration in polyhedron, to appear in Springer Undergraduater Texts in Mathematics.

[3] M. Beck, The partial-Fractiona method for counting solutions to integral linear systems, Discrete Comput. Geom. 32:437-446(2004).

[4] B. Bueler, A. Enge and K. Fukuda, Exact volume computation for polytopes: A practical study. In: Polytopes - Combinatorics and Computation, G. Kalai, and G. M. Ziegler, Eds., Birhäuser Verlag, Basel,(2000).

[5] C.de Boor and R.Devore, Approximation by smooth multivariate splines, Trans. Amer. Math. Soc.276(1983),775-788.

[6] C.de Boor and K.Höllig, B-splines from parallelepipeds, J. Analyse Math,42(1982/83).

[7] C.de Boor, K.Höllig and S.Riemenschneider, Box Splines, Springer-Verlag, New York,(1993).

[8] Michel Brion and Michèle Vergne, Residue formulae, vector partition functions and lattice points in rational polytopes, J. Amer. Math. Soc. 10(4)(1997),797-833.

[9] S.E. Caappell and J.L. Shaneson, Genera of algebraic varieties and counting of lattice points, Bull. A.M.S.30(1994),62-69.

[10] W.Dahmen, On multivariate B-splines, SIAM J. Numer. Anal. 17(1980),179-191.

[11] W.Dahmen and C.A. Micchelli, Translates of multivariate splines, Linear Algebra Appl. 52/53,(1983),217-234.

[12] W.Dahmen, and C.A. Micchelli, Recent progress in multivariate splines, in Approximation Theory IV(C. K. Chui, L. L. Schumaker, and J. Ward,Eds.), Academic, New York,1983,pp.27-121.

[13] W.Dahmen and C.A. Micchelli, On the solution of certain systems of partial difference equations and linear dependence of translates of box splines, Trans. Amer. Math. Soc. 292,(1985),305-320.

[14] W.Dahmen and C.A.Micchelli,On the local linear independence of translate of a box spline, Studia Math. 82,(1985),243-263. 
[15] W.Dahmen and C.A. Micchelli, The number of solutions to linear diophantine equations and multivariate splines, Trans. Amer. Math. Soc.308,(1988),509-532.

[16] Dinwoodie, I.,Stochastic simulation on integer constraint sets, SIAM J. Optimization 9(1999)53-61.

[17] Jesús A. De Loera and Bernd Sturmfels, Algebraic unimodular counting, Math. Program., Ser. B 96 (2003) 2, 183-203.

[18] J.-F. Romeuf, A polynomial algorithm for solving systems of two linear Diophantine equations. Theoret. Comput. Sci. 74 (1990), no. 3, 329-340.

[19] R. Stanley, Enumerative Combinctorics, Vol.1, Wadsworth, Belmont, Calif.,1986.

[20] R.Q. Jia, Multivariate discrete splines and linear diophantine equations, Trans. Amer. Math. Soc.340:1(1993),179-197.

[21] R.Q. Jia, Symmetric magic squares and multivariate splines,Linear Algebra Appl.,250(1997)69-103.

[22] C.A.Micchelli, On a numerically efficient method for computing multivariate Bsplines,in Multivariate Approximation Theory, W. Schempp, and K.Zeller, eds., Birkhäuser, Basel,(1979),211-248.

[23] Nijehuis, A. and Wilf, H., Representation of integers by linear forms in nonegative integers, J. Number Theory 4(1972),98-106.

[24] E. Ehrhart, Sur un problème de géométrie diophantienne linéaire II, J. Reine Angew. Math. 227(1967),25-49.

[25] J.M. Kantor and A. Khovanskii, Une application du Théorème de Riemann-Roch combinatoire au polynôme d'Ehrhar des polytopes entier de $R^{d}$, C.R. Acad. Sci. Paris, Series I 317 (1993),501-507.

[26] J. Pommersheim, Toric varieties, lattice points, and Dedekind sums, Math. Ann. 295(1993),1-24.

[27] Tiberiu Popoviciu, Asupra unei probleme de patitie a numerelor, Acad. Republicii Populare Romane, Filiala Cluj, Studii si cercetari stiintifice 4(1953),7-58.

[28] A. Rycerz, Conductors and the generalizaed problem of Frobenius, Discussiones Mathematicae 14(1994)15-20.

[29] Schmidt, J.R. and Bincer, A. The Kostant partition function for simple Lie algebras, J. Mathematical Physics 25(1984)2367-2373.

[30] A. Szenes and Michèle Vergne, Residue formulae for vector partitions and EulerMacLaurin sums,Adv. in Appl. Math. 30(2003), NO.1-2,295-342.

[31] Bernd Sturmfels, On vector partition functions, J. Combin. Theory Ser.A 72(2)(1995),302-309.

[32] Welsh, D. Approximate counting, in Surveys in Combinatorics, edited by R.A. Bailey, London Mathematical Society Lecture Notes, Vol.241,1997.

[33] R.H. Wang and Z.Q. Xu, Multivariate splines and lattice points in rational polytope, J. Comp. Appl. Math.,159 (2003) 149-159.

[34] Zhiqiang Xu, Discrete Truncated Powers, Volume of Convex Polytopes and Ehrhart Polynomials, http://arxiv.org/abs/math.CO/0505129.

[35] Zhu, Nan and Broughan, Kevin, On aggregating two linear Diophantine equations, Discrete Appl. Math, 82 (1998), no. 1-3, 231-246.

Institute of Computational Mathematics, Academy of Mathematics and System Science, Chinese Academy of Sciences, Beijing, China

E-mail address: xuzq@lsec.cc.ac.cn 